EPJ Web of Conferences 44, 01003 (2013)

DOI: $10.1051 /$ epjconf $/ 20134401003$

(c) Owned by the authors, published by EDP Sciences, 2013

\title{
Role of weighting and long-range connections on the dynamics of front propagation
}

\author{
K. Khelloufi, Y. Baara, N. Zekri* \\ Université des Sciences et de la Technologie d'Oran Mohamed Boudiaf, Département de Physique, LEPM, BP 1505 El \\ Mnaouer, Oran, Alger
}

\begin{abstract}
Percolation and non-equilibrium front propagation in a two-dimensional network modeling wildfire spread is studied. The model includes two long-range interactions; a deterministic and a probabilistic one induced by firebrand emission. It includes also a time weighting process. Three weight-dependent regimes were found previously; dynamical, static, and non-propagative regime [12]. In the absence of probabilistic interaction, the percolation threshold dependence on the weight does not depend on the deterministic interaction. The dynamical regime is found to belong to the dynamical percolation universality class and the static regime to the random deposition class. In the presence of probabilistic interactions, a minimum percolation threshold is found due to the scaling effects. The dynamical exponents belong to a new universality class.
\end{abstract}

\section{Introduction}

Phase transition, for equilibrium and non-equilibrium states (during growing processes or chemical reactions) $[1,2]$, ranks among the most fascinating phenomena in nature. Although it has been extensively studied for several decades, some problems regarding the universality and the dynamics of spreading at the phase transition remain open [6-8].

Percolation theory [3] is one of the models commonly used to describe phase transitions and has been applied to a wide range of phenomena, such as the displacement of oil in porous media, the propagation of forest fires, and epidemics [3-5].

Watts and Strogatz proposed in 1998 a random graph model: Small World Network (SWN) modeling the spread in social networks [5, 9-11]. It includes both randomly generated long-range connections (shortcuts) and a high connectivity [11].

Recently, the SWN model was adapted to forest fire spread [11-13]. In this model, in addition to the probabilistic long-range interactions representing the firebrands emission, deterministic long-range connections due to the ignition by flame radiations are introduced [12,13]. A weighting process appears due to the ignition energy of the combustible cell and the combustion time of the flame [12].

The effect of the long-range connections and the weighting process on both percolation transition and front dynamics is interesting to investigate. This is the aim of this work were the influence of flame radiation and firebrands on the percolation and front dynamics are examined.

\section{Model description}

The present model is a variant of the WattsStrogatz small-world network model built from a twodimensional $L$ sized lattice of square cells of size $a$ occupied with a probability $p$ and empty with a probability (1p).

The probabilistic long-range connection corresponds to the spotting process caused by firebrand's emission far from the cell. The spotting process [14] occurs under the action of wind and / or slope and when the brush and trees burn rapidly have a high plume of flame $(\approx 3 \mathrm{~m})[15]$. These incandescent particles or burning (for example: pieces of bark, pine needles, twigs, leaves and fragments of cork ...[15]) may give rise to some secondary fires "fire palace" from $100 \mathrm{~m}$ to $1 \mathrm{Km}$ away from the main fire front [15].

The average emission probability per cell $P_{0}$ is here fixed to unity, and the emission angle fixed as $\alpha=\pi / 3$ within the wind direction. The probability distribution of emission was found to be exponentially decreasing with distance $D[15,17]$ :

$\mathcal{P}=P_{0} \operatorname{Exp}\left(-\frac{D}{D_{0}}\right)$

$D_{0}$ is the characteristic emission distance. The number of effective firebrands depends on the size of the network where $[14,18]$ :

$\begin{cases}D_{0} \gg L & N_{f b}(t)=c N_{b s}(t) \frac{L}{D_{0}} \\ D_{0} \ll L & N_{f b}(t)=c N_{b s}(t)\end{cases}$

With $N_{b s}$ is the number of burning cells.

*nzekri@yahoo.com 
The deterministic long-range connection is characterized by two lengths $n_{x} a$ and $n_{y} a$ defining an influence zone of the flame radiation. The influence zone is circular $\left(n_{x}=n_{y}\right)$ in the isotropic case (i.e. no wind and flat terrain). It becomes elliptical and induces anisotropy in the presence of a terrain slope and/or wind speed [14]. The model network is shown in Fig. 1.

Each combustible cell $j$, in the influence zone of a burning cell $i$, receives a radiation flux power-law decreasing with the distance $\mathfrak{D}_{i j}[10,19-20]$. The power exponent was found to depend on the distance from the flame. Actually if the flame is point-like this exponent is 2 and becomes 1 close to a radiant panel. This exponent becomes 3 for asymptotically large distances for a cylindrical flame. Therefore, in the present model a power-law decreasing radiation flux with the distance is used

$$
P_{i j}=\int \frac{P_{0}}{\left(\left(\frac{\Delta n_{x_{i j}}}{n_{x}}\right)^{2}+\left(\frac{\Delta n_{y_{i j}}}{l n_{y}}\right)^{2}\right)^{\frac{\beta}{2}}} d t
$$

Here $\Delta n_{x_{i j}}$ and $\Delta n_{y_{i j}}$ are the jumps in directions $\mathrm{x}$ and $\mathrm{y}$ respectively, and the power-law exponent $\beta$ takes arbitrary values.

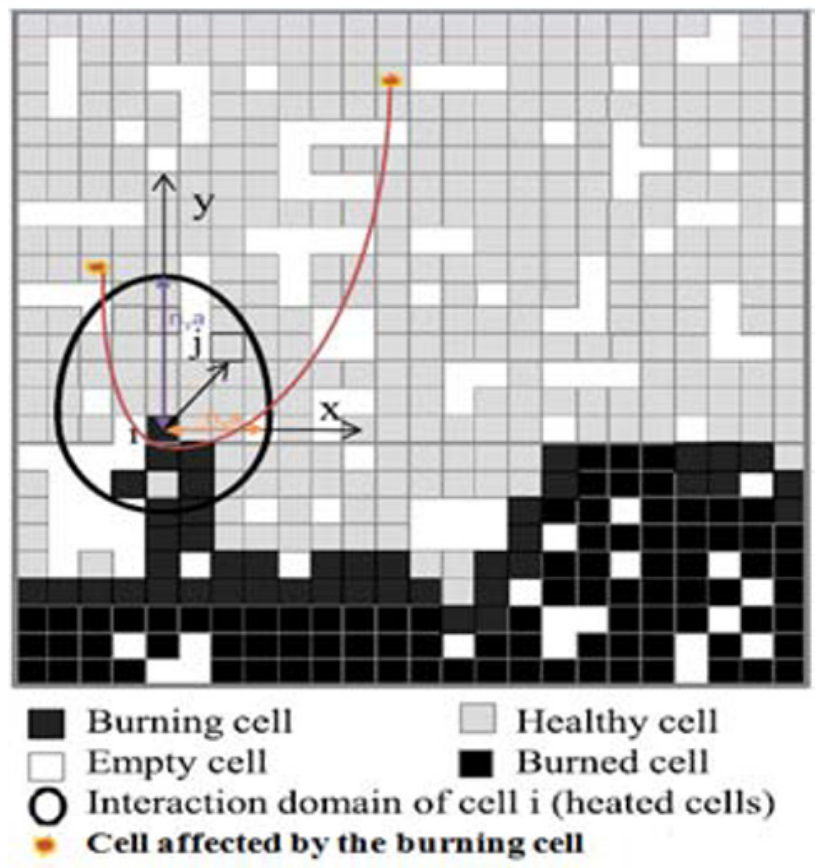

Fig. 1. The small world network model

The burning cell has a combustion time $t_{c}$, and the combustible cell is ignited when its energy reaches an ignition energy $E_{\text {ign. }}$. The propagation exhibits thus a weighting process corresponding to the ratio of the ignition energy to the energy produced by the burning cell during its residence time $E_{i g n} / P_{i j} t_{c}$ [12]. As this procedure depends on the distance to the burning cell $\mathfrak{D}_{i j}$, the weighting parameter $\mathrm{R}$ is defined as this ratio for cells located at the border of the influence zone

$$
R=\frac{E_{i g n}}{P_{0} t_{c}}
$$

For $\beta=2$, three propagation regimes were found depending on the values of $\mathrm{R}$; a dynamical regime for $R<R_{c}$, a static regime for $R=R_{c}$ and no propagation for $R>R_{c}$. $R_{c}$ is a critical weight where a burning cell can ignite only its nearest neighbors. It is thus defined as

$R_{c} \propto n_{y}^{\beta}$

\section{Results}

A square network of $300 \times 300$ cells is used. The cells are randomly occupied by combustible elements with a probability $p$. The percolation threshold $p_{c}$ corresponds statistically to the appearance of a cluster of burned cells connecting the opposite sides of the heterogeneous system. It is determined from the maximum burned mass fluctuations (as the disorder is larger at the phase transition). The results are averaged over 100 realizations which were found to be sufficient to avoid finite size effects [21] (The systems size used here ensures that $|p c(L)-p c(\infty)|$ is smaller than the error bar $(0.005)$ of $p c(L))$. In order to improve the statistics, the first horizontal line is initially ignited.

\subsection{Deterministic long-range effects}

The weight and deterministic long-range connections effects on the percolation threshold are now examined. For weights $\mathrm{R} \leq 1$ (saturation regime), the percolation threshold saturates at a minimum value independently of the exponent $\beta$ (Fig.2) [12].

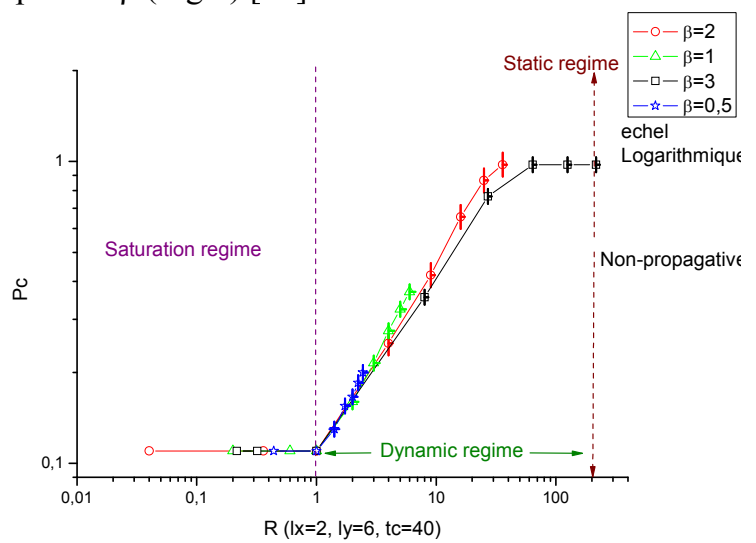

Fig. 2. The percolation threshold $\mathrm{p}_{\mathrm{c}}$ versus $R$ for $\mathrm{l}_{\mathrm{y}}=6 a$, $l_{x}=2 a$ and $\beta=0.5,1,2$ et 3

In this regime, the burning cell can ignite all cells located in its influence zone, and the number of burned cells will be independent of the weighting procedure. In the remaining dynamical regime $(1<\mathrm{R}<\mathrm{Rc})$, $\mathrm{pc}$ is independent of the interaction $(\beta)$ within the statistical errors. This confirms the universality of this phase transition. There is however a saturation effect close to $\mathrm{Rc}$ at $\mathrm{pc}=1$, for $\beta=$ 3 . As the system considered here is anisotropic, the limit $\mathrm{R}=\mathrm{Rc}$ makes the propagation nearly $1 \mathrm{D}$ and then $\mathrm{pc}$ reaches 1 . When $\beta$ increases this anisotropy is strengthened (see Eq.5), and starting from $\beta=3$ the $1 \mathrm{D}$ behavior is reached below Rc. For isotropic systems $\left(\mathrm{n}_{\mathrm{x}}=\mathrm{n}_{\mathrm{y}}\right)$, the percolation threshold reaches the limit $\mathrm{pc}=0.59$ (that of the square network [3]). 


\subsection{Probabilistic long-range effect:}

Let us now consider the spotting process. A secondary fire starts only in case of a connection between occupied cells. In this case the connection is effective. The percolation threshold is shown in fig.3. The percolation threshold exhibits a minimum at a given characteristic distance (here the minimum is observed for $\mathrm{D} 0=30 \mathrm{~m}$ ). This minimum is size dependent. It disappears for an infinite system. The behavior of pc is in quite agreement with the empirical Sander's formula for small world networks [16]

$$
4 C \phi=\frac{\left(P_{c}^{r r}-P_{c}^{p m}\right)^{\gamma}}{P_{c}^{p m}}
$$

Where $C$ is a constant, and $\gamma$ is a critical exponent determined from the universal scale of the correlation length: $\langle m\rangle=C\left|P-P_{c}\right|^{-\gamma}$

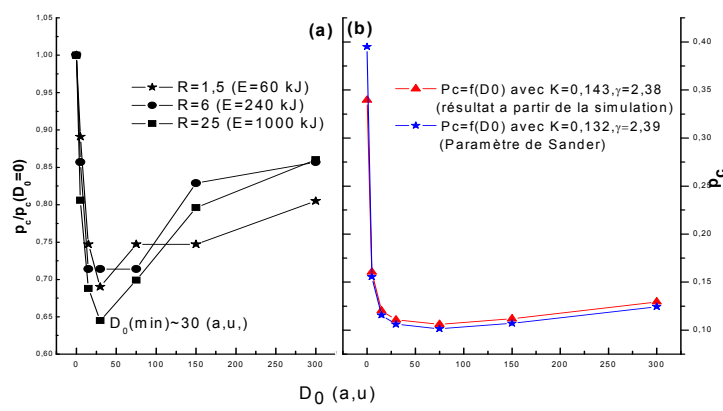

Fig. 3 : (a) $p_{c} / p_{c}\left(D_{0}=0\right)$ versus $\mathrm{D} 0$ for $\mathrm{ny}=6, \mathrm{nx}=2$ and $\mathrm{R}=1.5$, 6 et 25. (b) Comparison with Sander results.

The scaling behavior of the propagation time and burned mass fluctuations at pc show different power-law behaviors see fig. 4 .

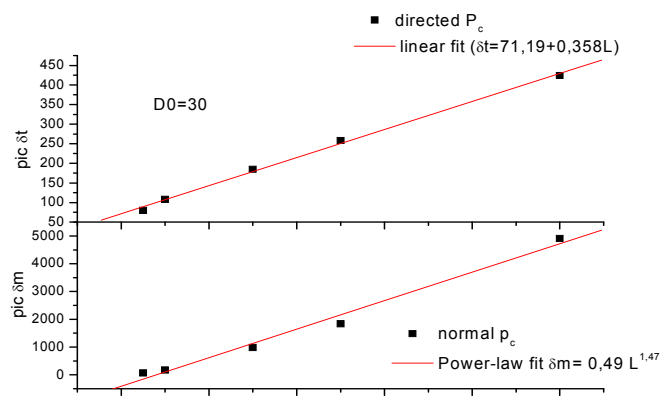

Fig. 4. Types of percolation: directed percolation $\delta \mathrm{t}=\mathrm{f}(\mathrm{L})$, normal $\delta \mathrm{m}=\mathrm{f}(\mathrm{L})$.

The propagation time fluctuations increase linearly

$\delta t=v_{p_{c}} L+b$

Where $\mathrm{v}_{\mathrm{p}_{\mathrm{c}}}$ is the rate of spread at pc.

The burned mass fluctuations increase as power law

$\delta \mathrm{m}=\mathrm{k} * \mathrm{~L}^{\sigma}$ with $\sigma=1.73$

This behavior indicates a power-law dependence of correlation length on that of time. This dynamical behavior will be investigated in the next section.

\subsection{Front dynamics}

The front dynamics is characterized by a set of nonequilibrium exponents. The aim here is to look for the universality class this model belongs on. We assume that the width of the fire interface is defined as $[6,8]$ :

$\mathrm{W}\left(\mathrm{t}, \mathrm{L}_{\mathrm{x}}\right)=\left\langle\left[\frac{1}{\mathrm{~L}_{\mathrm{x}}} \sum_{\mathrm{i}} \mathrm{y}_{\mathrm{i}}^{2}(\mathrm{t})-\left(\frac{1}{\mathrm{~L}_{\mathrm{x}}} \sum_{\mathrm{i}} \mathrm{y}_{\mathrm{i}}(\mathrm{t})\right)^{2}\right]^{1 / 2}\right\rangle$

Where $y_{i}(t)$, is the maximum fire position in the wind (y) direction, $\mathrm{Lx}$ the lateral system size and $\langle\ldots\rangle$ denotes the average over realizations. Below the correlation time $\tau$, the interface width is expected to behave as $\alpha t^{\beta}$, where $\beta$ is the growth exponent. For $t>\tau$, the interface width saturates and scales as $\mathrm{W}(\mathrm{t}>\tau, L x) \propto \mathrm{L}_{\mathrm{x}}^{\alpha}$, where $\alpha$ is the roughness exponent (also called Hurst exponent) [8].

The correlation time also scales as $\tau \propto \mathrm{L}_{\mathrm{x}}^{\mathrm{z}}$ ( $\mathrm{z}$ is the socalled dynamic exponent). These exponents are not independent $(\beta=\alpha / z)$. This scaling picture is known as Family-Vicsek scaling ansatz [22].

For finite systems and point ignition, the mean square of spreading distance $\left(\mathrm{y}_{\mathrm{i}}(\mathrm{t})\right)$ from the origin scales as [6]:

$\Re^{2}=\frac{1}{N(t)}\left\langle\sum_{i} y_{i}^{2}(t)\right\rangle \propto t^{z}$

Where $z=2 / Z$ is the dynamic exponent.

From a previous paper-burning experiment of Zhang et al. [23] the roughness exponent is 0.71 . Later on, Provatas et al. used a phase-field model to describe flame propagation in random continuous media [24]. They obtained $\alpha=1 / 2$ and $\beta=1 / 3$. Discrepancies between model and experiments were explained by the correlated distribution of fibers within paper.

Roughening exponents were determined and found consistent with the Kardar-Parisi-Zhang (KPZ) universality class [7]. However, the propagation in continuous media does not take into account neither long-range interactions nor the weighting process.

From the present work and deterministic long-range interaction, we find $\beta=0.218 \pm 0.02, \alpha=0.3 \pm 0.03$ and $z=$ $0.976 \pm 0.08$ in the dynamic region $(\mathrm{R}=1)$, not far from the dynamic exponent characterizing the dynamical percolation class, with $\mathrm{z}=1.13$ [6]. In the static region $(\mathrm{R}=$ $\mathrm{Rc})$ the percolation of the fire seems to be onedimensional and the disorder is responsible of stopping the fire so that the system belongs to the random deposition universality class.

Let us now examine the front dynamical exponents in the case of the spotting process. In the static regime the dynamics of propagation belongs to random deposition universality class (the spotting process seems to have no influence on the front dynamics in this case).

For $\mathrm{R}=1$, the exponents change, with $\mathrm{z}=0.75+-0.04$ indicating a super-diffusive behavior. This is mainly caused by the contribution of the secondary fire fronts. This behavior belongs to a new universality class "Directed Percolation Depinning class" [8].

\begin{tabular}{ccccc}
\hline Exponent & Deterministic interaction & Probabilistic interaction \\
\hline The weigh & $\mathrm{R}=1$ & $\mathrm{R}=\mathrm{R}_{\mathrm{c}}$ & $\mathrm{R}=1$ & $\mathrm{R}=\mathrm{R}_{\mathrm{c}}$ \\
\hline
\end{tabular}




\begin{tabular}{ccccc}
\hline $\boldsymbol{\alpha}$ & $0.30 \pm 0.03$ & $\infty$ & $0.59 \pm 0.03^{*}$ & $0.58 \pm 0.04^{*}$ \\
$\boldsymbol{\beta}$ & $0.218 \pm 0.02$ & $0.627 \pm 0.03$ & $0.79 \pm 0.04$ & $0.49 \pm 0.03$ \\
$\mathbf{Z}$ & $0.976 \pm 0.08$ & $1.092 \pm 0.04$ & $0.75 \pm 0.04$ & $1.2 \pm 0.04$ \\
\hline
\end{tabular}

* obtained using $Z=\alpha / \beta[8]$.

Tab 1. Comparison of the scaling interface exponents in the dynamic and static regimes for the two type of the long-range interaction "probabilistic \& deterministic"

\section{Conclusion}

We have used a variant of the Watts and Strogatz small world network model to study the effect of weight and long range connections on the percolation and front dynamic of forest fire spread. The main results are summarized below:

- The weight dependence of percolation threshold is not affected by the interaction.

- A minimum percolation threshold is observed in the presence of the firebrands. This is due to size effect. And is in quite agreement with the empirical formula of sanders.

- In the static regime, the model belongs to the random deposition universality class.

- In the dynamic regime, the system belongs to the dynamical percolation universality class in the absence of probabilistic long-range interactions. In the presence of these interactions, a new set of exponents appears leading to DPD universality class.

\section{Acknowledgements}

Mr. A. Miguel. Muñoz, is gratefully acknowledged for insightful discussions on this problem.

\section{Reference}

1. M.Plishke and B.Bergersen, Equilibrium statistical physics (World Scientific, Singapore) 1994.

2. R.Balescu, Statistical Physics out of equilibrium (Imperial College Press, London) 1997.

3. D.Stauffer and A.Aharony, Introduction to percolation theory (Taylor and Francis, London) 1992.

4. P.J.Flory, J.Am.Chem.Soc. 63 (1941) 3083.

5. M.E.J.Newman, Small Worlds (Princeton University Press, Princeton) 1999.

6. G.Odor .rev. Mod. Phys. 76 (2004) 663.

7. M.Kardar, G.Parisi, Y.-C.Zhang. Rev. Lett. 56 (1986) 889.

8. A.L.Barabasi and H.E.Stanly, Fractal concepts in surface growth (Cambridge university Press) 1995.

9. F.Comellasa, M.Sampelsb; Deterministic small-world networks Physica A 309 (2002) 231-235.

10. J.K. Adou, Y. Billaud, D.A. Brou, J.-P. Clerc, J.-L. Consalvi, A. Fuentes, A. Kaiss, F. Nmira, B. Porterie, L. Zekri, N. Zekri, Ecological Modelling 221 (2010) 1463-1471.

11. D.J. Watts, S.H. Strogatz, Collective dynamics of 'small-world' networks, Nature 393 (1998) 440-442.

12. N. Zekri , K. Khelloufi, L. Zekri, B. Porterie, A. Kaiss et J.P.Clerc, Phys.Lett. A 376 (2012) 2522.
13. B. Porterie, A. Kaiss, J.P. Clerc, L. Zekri, and N. Zekri, Appl. Phys. Lett. 93 (2008) 204101.

14. B. Porterie, N. Zekri, J.P. Clerc, J.C. Loraud, Combustion and Flame 149 (2007) 63-78.

15. J.L.Consalvi, P.Mindykowski, J.P.Vantelonb, B.Porterie. Fire Safety Journal 46 (2011) 48-55.

16. L.M.Sander, C.P. Warren, I.M.Sokolov, C.Simon, J. Koopman, Math.Biosc. 180 (2002) 293.

17. www.ffp.csiro.au/nfm//fbm/vesta/spotfire.html.

18. N.Sardoy, J.L.Consalvi, B.Porterie, J.C.Loraud, JITH2005, Tangiers (2005).

19. Y.Billau, A.Kaiss, J.-L.Consalvi and B.Porterie, Int.J.Therm.Science, 50, (2011) 2.

20. K.S. Mudan, Thermal radiation hazards from hydrocarbon pool fires Prog. Energy. Combust. Sci. 10 (1984) 59.

21. N.Zekri, B.Porterie, J.P.Clerc, J.C.Loraud, phys. Rev. E 71 (2005) 046121.

22. F.Family, T.Viscek (Eds.), Dynamics of fractal surfaces, World scientific, Singapore (1991).

23. J.Zhang, Y,-C,Zhang, P,Aleston, M.T.Levinsen, Phys. A 189 (1992) 383.

24. N.Provatas, T.Ala-Nissila, M.Grant, K.R.Elder, L.Piché, Phys. Rev. E 51 (1995) 4232. 